\title{
High-quality genome sequence of the radioresistant bacterium Deinococcus ficus KS 0460
}

Vera Y. Matrosova ${ }^{1,2+}$, Elena K. Gaidamakova ${ }^{1,2+}$, Kira S. Makarova ${ }^{3}$, Olga Grichenko ${ }^{1,2}$, Polina Klimenkova ${ }^{1,2}$, Robert P. Volpe ${ }^{1,2}$, Rok Tkavc ${ }^{1,2}$, Gözen Ertem', Isabel H. Conze ${ }^{1,4}$, Evelyne Brambilla ${ }^{5}$, Marcel Huntemann ${ }^{6}$, Alicia Clum ${ }^{6}$, Manoj Pillay ${ }^{6}$, Krishnaveni Palaniappan ${ }^{6}$, Neha Varghese $^{6}$, Natalia Mikhailova $^{6}$, Dimitrios Stamatis ${ }^{6}$, TBK Reddy ${ }^{6}$, Chris Daum ${ }^{6}$, Nicole Shapiro ${ }^{6}$, Natalia Ivanova ${ }^{6}$, Nikos Kyrpides ${ }^{6}$, Tanja Woyke $^{6}$, Hajnalka Daligault $^{7}$,

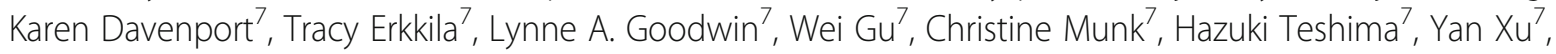
Patrick Chain ${ }^{7}$, Michael Woolbert ${ }^{1,2}$, Nina Gunde-Cimerman ${ }^{8}$, Yuri I. Wolf ${ }^{3}$, Tine Grebenc ${ }^{9}$, Cene Gostinčar ${ }^{8}$ and Michael J. Daly ${ }^{1 *}$

\begin{abstract}
The genetic platforms of Deinococcus species remain the only systems in which massive ionizing radiation (IR)-induced genome damage can be investigated in vivo at exposures commensurate with cellular survival. We report the whole genome sequence of the extremely IR-resistant rod-shaped bacterium Deinococcus ficus KS 0460 and its phenotypic characterization. Deinococcus ficus KS 0460 has been studied since 1987, first under the name Deinobacter grandis, then Deinococcus grandis. The D. ficus KS 0460 genome consists of a 4.019 Mbp sequence (69.7\% GC content and 3894 predicted genes) divided into six genome partitions, five of which are confirmed to be circular. Circularity was determined manually by mate pair linkage. Approximately $76 \%$ of the predicted proteins contained identifiable Pfam domains and $72 \%$ were assigned to COGs. Of all D. ficus KS 0460 proteins, 79\% and $70 \%$ had homologues in Deinococcus radiodurans ATCC BAA-816 and Deinococcus geothermalis DSM 11300, respectively. The most striking differences between D. ficus KS 0460 and D. radiodurans BAA-816 identified by the comparison of the KEGG pathways were as follows: (i) $D$. ficus lacks nine enzymes of purine degradation present in $D$. radiodurans, and (ii) $D$. ficus contains eight enzymes involved in nitrogen metabolism, including nitrate and nitrite reductases, that $D$. radiodurans lacks. Moreover, genes previously considered to be important to IR resistance are missing in D. ficus KS 0460, namely, for the Mn-transporter nramp, and proteins DdrF, DdrJ and DdrK, all of which are also missing in Deinococcus deserti. Otherwise, D. ficus KS 0460 exemplifies the Deinococcus lineage.
\end{abstract}

Keywords: Deinococcus-Thermus, Deinococcaceae, Deinococcus ficus, Radiation-resistant, Rod-shaped, Phenotype characterization, Genome analysis, Phylogenetic analysis

\footnotetext{
* Correspondence: michael.daly@usuhs.edu

${ }^{\dagger}$ Equal contributors

'Uniformed Services University of the Health Sciences, School of Medicine,

Bethesda, MD, USA

Full list of author information is available at the end of the article
} 


\section{Introduction}

Species of the genus Deinococcus have been studied for their extreme IR resistance since the isolation of Deinococcus radiodurans in 1956 [1]. Since then, many other species of the same genus have been isolated. The current number of recognized Deinococcus species is greater than 50 while there are more than 300 non-redundant 16S rRNA sequences of the family Deinococcaceae in the ARB project database [2]. Apart from Deinococcus ficus KS 0460, only a few other representatives have been studied in detail for their oxidative-stress resistance mechanisms: D. radiodurans, Deinococcus geothermalis and Deinococcus deserti [3]. The picture that has emerged for the life cycle of most Deinococcus species is one comprised of a cellreplication phase that requires nutrient-rich conditions, such as in the gut of an animal, followed by release, drying and dispersal [1]. Desiccated deinococci can endure for years, and, if blown by winds through the atmosphere, are expected to survive and land worldwide. As reported, some deinococci become encased in ice, and some entombed in dry desert soils. High temperatures also are not an obstacle to the survival of some deinococcal species. D. geothermalis and Deinococcus murrayi were originally isolated from hot springs in Italy and Portugal, respectively [1]. The prospects of harnessing the protective systems of $D$. radiodurans for practical purposes are now being realized.

The complete genome sequence presented here is for $D$. ficus KS 0460, originally named Deinobacter grandis KS 0460, isolated in 1987 from feces of an Asian elephant (Elephas maximus) raised in the Ueno Zoological Garden, Tokyo, Japan (Table 1) [4]. Later, Deinobacter grandis was renamed Deinococcus grandis [5]. Strain KS 0460 was acquired by USUHS from the originating laboratory in 1988 by Kenneth W. Minton and has been the subject of study here ever since. As a candidate for bioremediation of radioactive DOE waste sites [6] and a target of study for

Table 1 Classification and general features of Deinococcus ficus KS 0460 according to MIGS recommendations [49]

\begin{tabular}{|c|c|c|c|}
\hline MIGS ID & Property & Term & Evidence code $^{a}$ \\
\hline & Classification & Domain Bacteria & TAS [50] \\
\hline & & Phylum Deinococcus-Thermus & $\operatorname{TAS}[51,52]$ \\
\hline & & Class Deinococci & $\operatorname{TAS}[53,54]$ \\
\hline & & Order Deinococcales & TAS [5] \\
\hline & & Family Deinococcaceae & TAS $[5,55]$ \\
\hline & & Genus Deinococcus & $\operatorname{TAS}[5,55]$ \\
\hline & & Species Deinococcus ficus & $\operatorname{TAS}[4,9]$ \\
\hline & & Strain: KS 0460 & \\
\hline & Gram stain & Variable & $\operatorname{TAS}[4,9]$ \\
\hline & Cell shape & Rod & $\operatorname{TAS}[4,9]$ \\
\hline & Motility & Non-motile & $\operatorname{TAS}[4,9]$ \\
\hline & Sporulation & None & $\operatorname{TAS}[4,9]$ \\
\hline & Temperature range & Mesophile & $\operatorname{TAS}[4,9]$ \\
\hline & Optimum temperature & $30-37^{\circ} \mathrm{C}$ & $\operatorname{TAS}[4,9]$ \\
\hline & pH range; Optimum & e.g. 5.5-10.0; 7.0 & $\operatorname{TAS}[4,9]$ \\
\hline & Carbon source & Glucose, fructose & TAS [9] \\
\hline MIGS-6 & Habitat & Elephas maximus feces & TAS [4] \\
\hline MIGS-6.3 & Salinity & $1 \% \mathrm{NaCl}(w / v)$ & TAS [4] \\
\hline MIGS-22 & Oxygen requirement & Aerobic & TAS [4] \\
\hline MIGS-15 & Biotic relationship & Free-living & NAS \\
\hline MIGS-14 & Pathogenicity & Non-pathogen & NAS \\
\hline MIGS-4 & Geographic location & Tokyo/Japan & TAS [4] \\
\hline MIGS-5 & Sample collection & 1987 & TAS [4] \\
\hline MIGS-4.1 & Latitude & Non reported & \\
\hline MIGS-4.2 & Longitude & Non reported & \\
\hline MIGS-4.4 & Altitude & Non reported & \\
\hline
\end{tabular}

aEvidence codes - IDA: Inferred from Direct Assay; TAS: Traceable Author Statement (i.e., a direct report exists in the literature); NAS: Non-traceable Author Statement (i.e., not directly observed for the living, isolated sample, but based on a generally accepted property for the species, or anecdotal evidence). These evidence codes are from the Gene Ontology project [56] 
DNA repair [7], D. ficus KS 0460 was chosen for whole genome sequencing. The $D$. ficus KS 0460 genome now adds to the growing number of sequenced Deinococcus species needed to decipher the complex extreme IR resistance phenotype. To date, a genetic explanation for the complex survival tactics of deinococci has not been provided by comparative genomics or transcriptomics [8].

\section{Organism information}

\section{Classification and features}

In a chemotaxonomic study published in 1987, an isolate (strain KS 0460) from $\gamma$-irradiated feces of an Asian elephant yielded an IR-resistant bacterium with a wall structure, cellular fatty acid composition, and GC content typical of members of the genus Deinococcus [4]. However, strain KS 0460 was rod-shaped and grew as pinkpigmented colonies, whereas most other deinococci grow as diplococci/tetracocci and yield red colonies. The original isolate was named Deinobacter grandis, but was later renamed Deinococcus grandis based on its close phylogenetic relationship (16S rRNA sequences) with deinococci [5]. Strain KS 0460 was subsequently included in experimental IR survival studies together with other Deinococcus species, where it was referred to as grandis [7]. Our $16 \mathrm{~S}$ rRNA phylogenetic analysis confirms that strain KS 0460 belongs to the genus Deinococcus, most closely related to the type strain of Deinococcus ficus DSM 19119 (also referred to as CC-FR2-10) (Fig. 1).

Consistent with the original description of D. ficus KS 0460 , the rod-shaped cells are 0.5 to $1.2 \mu \mathrm{m}$ by 1.5 to $4.0 \mu \mathrm{m}$ (Fig. 2a) and grow as pink colonies [4, 9]. D. ficus KS 0460 was shown to have a $D_{10}$ of approximately $7 \mathrm{kGy}(\mathrm{Co}-60)$ (Fig. 2b) and is capable of growth under chronic $\gamma$-irradiation at $62 \mathrm{~Gy} / \mathrm{h}$ (Cs-137) (Fig. 2c). The cells are aerobic, incapable of growth under anaerobic conditions on rich medium, irrespective of the presence or absence of chronic IR (Fig. 2c). The general structure of the D. ficus KS 0460 genome was analyzed by PFGE of genomic DNA prepared from embedded cells. The plugs containing digested cells were exposed to 200 Gy prior to electrophoresis, a dose gauged in vitro to induce approximately 1 DNA double strand break per chromosome in the range $0.5-2 \mathrm{Mbp}$ [10]. Fig. 2d shows the presence of the five largest genomic partitions: main chromosome ( 2.8 Mbp), 3 megaplasmids $(\sim 500 \mathrm{~kb}$, $\sim 400 \mathrm{~kb}$ and $\sim 200 \mathrm{kbp})$ and one plasmid ( 98 kbp), predicting a genome size $\sim 4.0 \mathrm{Mbp}$. We did not observe the smallest genome partition $(0.007 \mathrm{Mbp})$ by PFGE. The growth characteristics of $D$. ficus KS 0460 in liquid culture at 32 and $37{ }^{\circ} \mathrm{C}$ (Fig. 2e) are very similar to $D$.

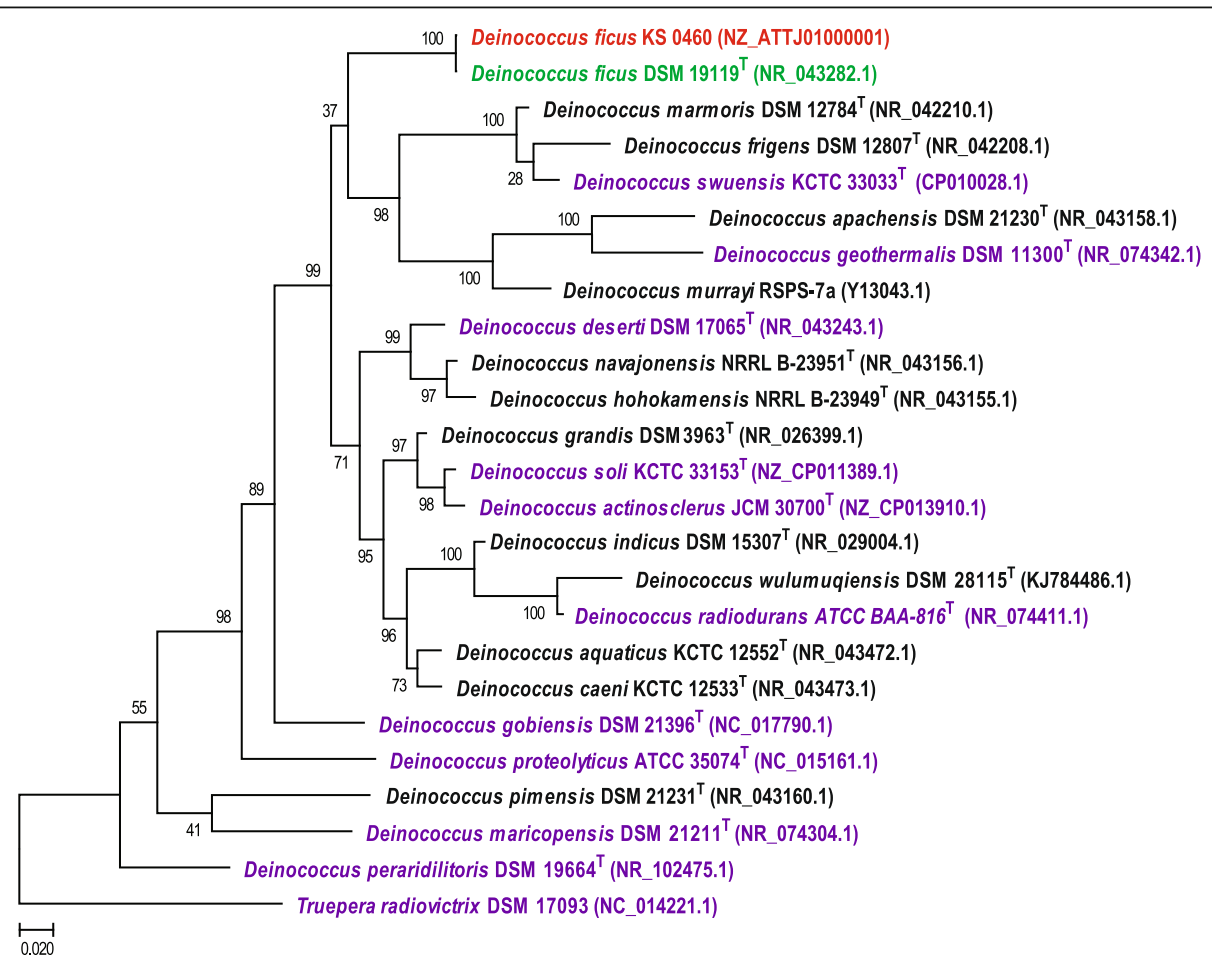

Fig. $116 S$ rRNA phylogenetic tree of the Deinococcus genus. The multiple alignment of $16 S$ rRNA sequences was constructed using MUSCLE program [58] with default parameters. The maximum-likelihood phylogenetic tree was reconstructed using the FastTree program [59], with GTR substitution matrix and gamma-distributed evolutionary rates. The same program was used to compute bootstrap values. Truepera radiovictrix was chosen as an outgroup. D. ficus KS 0460 is marked in red, D. ficus DSM 19119/CC-FR2-10 [9] - in green, completely sequenced according to NCBI genomes - in purple 
A

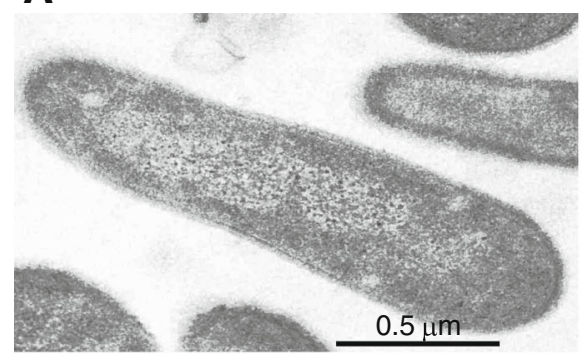

C

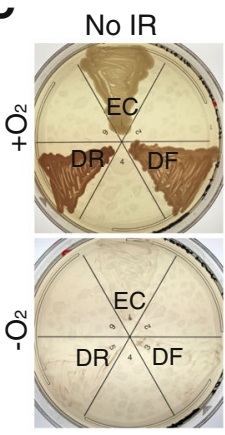

E

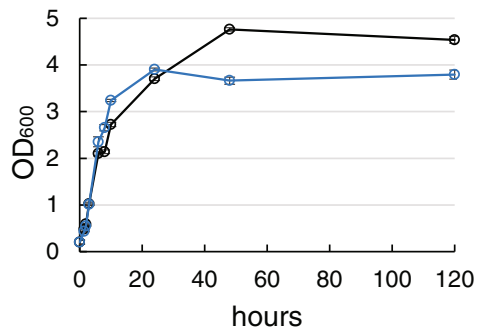

G

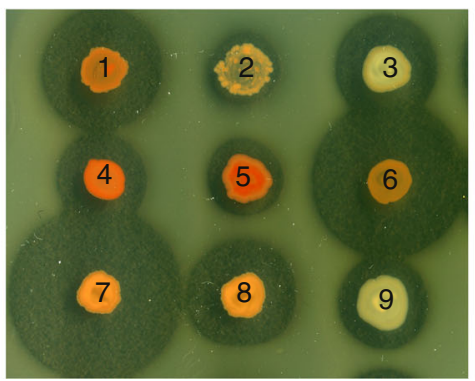

B

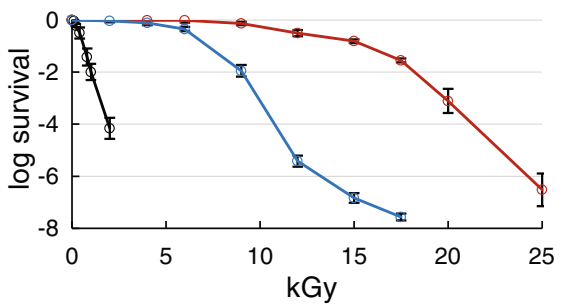

D
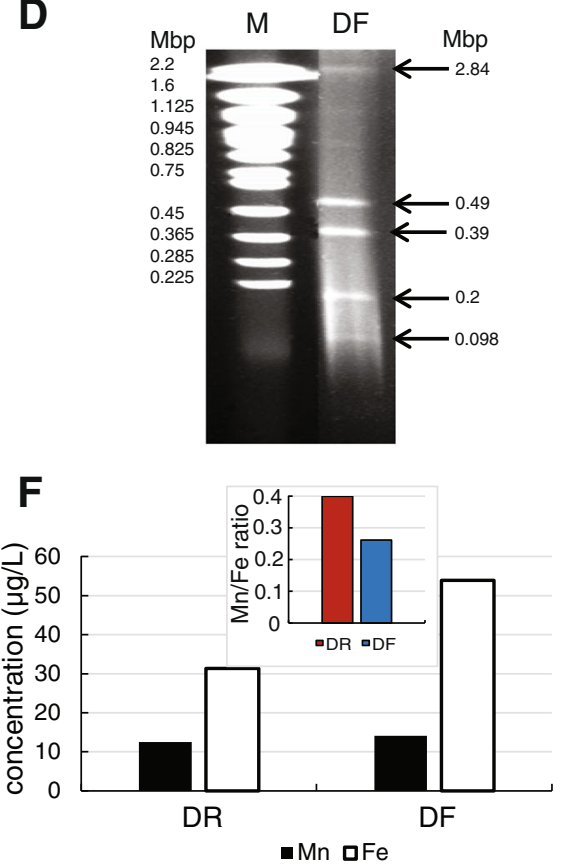

H

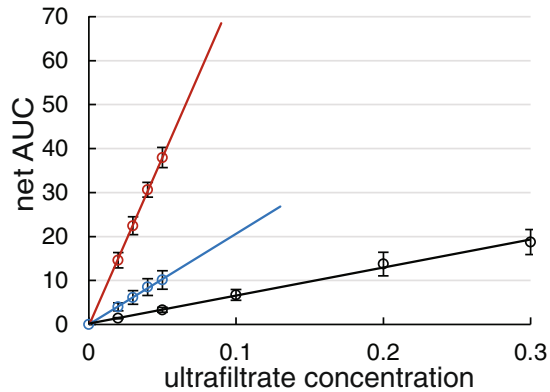

Fig. 2 Deinococcus ficus KS 0460 (EXB L-1957) phenotype. a Transmission electron micrograph. D. ficus grown in TGY, early-stationary phase. b Survival of D. radiodurans BAA-816 (red), D. ficus (blue), and E. coli (strain K-12, MG1655) (black) exposed to acute IR. The indicated strains were inoculated in liquid TGY and grown to $\mathrm{OD}_{600} \sim 0.9$. Cells were then irradiated on ice with Co-60. c D. ficus is an aerobe capable of growth under $62 \mathrm{~Gy} / \mathrm{h}$. DR, D. radiodurans; DF, D. ficus; EC, E. coli. d PFGE of genome partitions in a $0.9 \%$ agarose gel. PFGE conditions: $0.5 \times \mathrm{TBE}, 6 \mathrm{~V} / \mathrm{cm}$ with a 10 to $100 \mathrm{~s}$ switch time ramp at an included angle of $120^{\circ}, 14^{\circ} \mathrm{C}, 18 \mathrm{~h}$. M, marker S. cerevisiae YNN (BioRad). e Growth curves at $37^{\circ} \mathrm{C}$ (blue) and $32^{\circ} \mathrm{C}$ (black) in TGY medium. $\mathbf{f}$ ICP-MS on Mn and Fe content of D. radiodurans BAA-816 and D. ficus. Inset: Mn/Fe ratios. $\mathbf{g}$ Protease secretion assay. Halos indicate activity of proteases [60]. Strains: 1. D. radiodurans BAA-816, 2. D. geothermalis DSM 11300, 3. D. ficus KS 0460, 4. D. murrayi (MD591), 5. D. radiopugnans (MD567), 6. D. radiodurans (MD878, SX-108-7B-1, [61]), 7. D. proteolyticus (MD568), 8. D. proteolyticus (MD628, [62]), and 9. D. proteolyticus (MD869). h Antioxidant capacities of D. radiodurans BAA-816 (red), D. ficus (blue), and E. coli (strain K-12, MG1655) (black) ultrafiltrates assessed by antioxidant assay as described previously $[63,64]$. Net AUC is an integrative value of a total fluorescence during antioxidant reaction in the presence of ultrafiltrates 
radiodurans [11]. It is unknown if strain D. ficus KS 0460 is genetically tractable because the cells are naturally resistant to the antibiotics tetracycline, chloramphenicol and kanamycin at concentrations needed to select for plasmids and integration vectors designed for D. radiodurans [12] (data not shown). D. ficus KS 0460, like other deinococci, accumulate high concentrations of $\mathrm{Mn}^{2+}$ (Fig. 2f) [7, 13]. Bacterial $\mathrm{Mn}^{2+}$ accumulation was previously shown to be important to extreme IR resistance, mediated by the $\mathrm{Mn}$ transport gene nramp and ABC-type Mn-transporter gene [14]. We also showed that $D$. ficus KS 0460 produces proteases, as detected in a protease secretion assay on an indicator plate containing skimmed milk (Fig. 2g). For example, in D. radiodurans, the products of proteases - peptides - form $\mathrm{Mn}^{2+}$-binding ligands of Deinococcus $\mathrm{Mn}$ antioxidants, which protect proteins from IR-induced ROS, superoxide in particular $[8,13,15]$. Finally, we show that $D$. ficus KS 0460 cells have a high intracellular antioxidant capacity (Fig. 2h), which is a strong molecular correlate for IR resistance $[1,11]$.

\section{Extended feature descriptions}

$16 \mathrm{~S}$ rDNA gene phylogenetic analysis was based on sequences from 22 type strains of genus Deinococcus including ten from completely sequenced genomes, and two from Deinococcus ficus strains KS 0460 and DSM 19119; and Truepera radiovictrix DSM 17093, the distinct species shown to be an outgroup to the Deinococcus genus [16]. The maximum-likelihood phylogenetic trees were reconstructed using two approaches: (i) the FastTree program [17], with GTR substitution matrix and gammadistributed evolutionary rates and maximum-likelihood algorithm; and (ii) PHYML program with the same parameters (Fig. 1 and Additional file 1: Figure S1) [18]. Both $D$. ficus strains, as expected, group together, but the position of this pair in both trees is poorly resolved (37 support value for FastTree method and 44 for PHYML method) potentially because of the long branch of this clade. In both trees, however, the $D$. ficus clade confidently groups deep in the Deinococcus tree within the branch with $D$. gobiensis as a sister clade.

\section{Genome sequencing information \\ Genome project history}

Deinococcus ficus KS 0460 was obtained from the Oyaizu laboratory and was entered into the Daly strain collection at USUHS on November 18, 1997. The strain was submitted to the EX Culture Collection, Mycosmo, Slovenia, on December 29, 2016 and was issued an accession number EXB L-1957. The genome of D. ficus KS 0460 was sequenced at the JGI. The project was initiated in 2009, the genome was released on August 26, 2012 as "Deinococcus sp. 2009". The genome of D. ficus KS 0460 has the status of an improved high-quality draft. The genome assembly and annotation can be accessed through the JGI genome portal [19] and also GenBank [20]. The genome is considered to be nearcomplete. The search for bacterial Benchmarking Universal Single-Copy Orthologs [21] found a comparable number of orthologs in D. ficus KS 0460 and in ten complete Deinococcus species genomes. Furthermore, of the 875 genes representing the core genome of the same ten complete Deinococcus species as determined by the GET_HOMOLOGUES pipeline [22], only five genes were missing from $D$. ficus KS 0460.

\section{Growth conditions and genomic DNA preparation}

D. ficus KS 0460 was recovered from a glycerol frozen stock on TGY solid rich medium (1\% bactotryptone, $0.1 \%$ glucose, and $0.5 \%$ yeast extract, $1.5 \% \mathrm{w} / v$ bacto agar) ( 3 days, $32{ }^{\circ} \mathrm{C}$ ) with following inoculation of $25 \mathrm{ml}$ TGY medium. The culture was grown up to $\mathrm{OD}_{600} \sim 0.9$. Subsequently, $19 \mathrm{ml}$ were used to inoculate $2 \mathrm{~L}$ of TGY medium and the culture was grown at $32{ }^{\circ} \mathrm{C}$, overnight in aerated conditions in a shaker incubator (200 rpm). The cells were harvested at $\mathrm{OD}_{600} \sim 1.6$. The DNA was isolated from a cell pellet (5.6 g) using Jetflex Genomic DNA Purification Kit (GENOMED, Germany). The final DNA concentration was $80 \mu \mathrm{g} \mathrm{ml}^{-1}$, in a volume of $800 \mu \mathrm{l}$. The DNA was RNA free and passed quality control.

\section{Genome sequencing and assembly}

The draft genome of D. ficus KS 0460 was generated at the JGI using Illumina data (Table 2) [23]. Two pairedend Illumina libraries were constructed, one short-insert paired-end library (the length of paired-end reads was $150 \mathrm{bp}$ for the short insert library, average insert size of $222+/-50 \mathrm{bp}$ ), which generated 16,857,646 reads, and one long-insert library (average insert size of $7272+$ /729 bp), which generated 24,172,042 reads totaling 4946 Mbp of Illumina data. All general aspects of library construction and sequencing were performed at the JGI [19]. The initial draft assembly contained 9 contigs in 8 scaffolds. The initial draft data was assembled with Allpaths, version r38445, and the consensus was computationally shredded into $10 \mathrm{kbp}$ overlapping fake reads (shreds). The Illumina draft data was also assembled with Velvet, version 1.1.05 [24], and the consensus sequences were computationally shredded into $1.5 \mathrm{kbp}$ overlapping fake reads. The Illumina draft data was assembled again with Velvet using the shreds from the first Velvet assembly to guide the next assembly. The consensus from the second Velvet assembly was shredded into $1.5 \mathrm{kbp}$ overlapping fake reads. The fake reads from the Allpaths assembly, both Velvet assemblies, and a subset of the Illumina CLIP paired-end reads were finally assembled using parallel phrap, version 4.24 (High Performance Software, LLC). Possible misassemblies 
Table 2 Project information

\begin{tabular}{|c|c|c|}
\hline MIGS ID & Property & Term \\
\hline MIGS 31 & Finishing quality & High-Quality Draft \\
\hline MIGS-28 & Libraries used & $\begin{array}{l}\text { Illumina Standard (short } \\
\text { insert paired-end) and } \\
\text { Illumina CLIP (long insert } \\
\text { paired-end) }\end{array}$ \\
\hline MIGS 29 & Sequencing platforms & $\begin{array}{l}\text { Illumina HiSeq } 2000 \text { (CLIP } \\
\text { library); Illumina HiSeq } 2000 \\
\text { (Standard library); PacBio }\end{array}$ \\
\hline MIGS 31.2 & Fold coverage & $1237 x$ \\
\hline MIGS 30 & Assemblers & $\begin{array}{l}\text { Allpaths } r 38445 \text { and } \\
\text { Velvet 1.1.05 }\end{array}$ \\
\hline \multirow[t]{6}{*}{ MIGS 32} & Gene calling method & $\begin{array}{l}\text { Prodigal within JGl } \\
\text { Prokaryotic Automatic } \\
\text { Annotation Pipeline }\end{array}$ \\
\hline & Locus Tag & DEINO \\
\hline & Genbank ID & ATTJ00000000.1 \\
\hline & GenBank Date of Release & 07/09/2013 \\
\hline & GOLD ID & Gp0007971 \\
\hline & BIOPROJECT & PRJNA157079 \\
\hline \multirow[t]{2}{*}{ MIGS 13} & Source Material Identifier & EXB L-1957 \\
\hline & Project relevance & $\begin{array}{l}\text { DNA repair mechanisms, } \\
\text { bioremediation }\end{array}$ \\
\hline
\end{tabular}

were corrected with manual editing in Consed [25-27]. Gap closure was accomplished using repeat resolution software [Wei $\mathrm{Gu}$, unpublished], and sequencing of bridging PCR fragments with Sanger and/or PacBio technologies [Cliff Han, unpublished]. A total of 21 PCR PacBio consensus sequences were completed to close gaps and to raise the quality of the final sequence.

\section{Genome annotation}

The genome sequence was annotated using the JGI Prokaryotic Automatic Annotation Pipeline [28] and further reviewed using the Integrated Microbial Genomes Expert Review platform [29]. Genes were predicted using Prodigal [30], followed by a round of manual curation using the JGI GenePRIMP pipeline [31]. The genome sequence was analyzed and released publicly through the Integrated Microbial Genomes platform [32]. BLASTClust was used to identify internal clusters with thresholds of $70 \%$ covered length and $30 \%$ sequence identity [33]. SignalP [34] and TMHMM [35] were used to predict signal peptides and transmembrane helices, respectively.

\section{Genome properties}

The D. ficus KS 0460 genome consists of a 4,019,382 bp sequence which represents six genome partitions: 2.84, 0.49, $0.39,0.20,0.098$ and $0.007 \mathrm{Mbp}$ (Table 3), consistent with PFGE (Fig. 2d); note, the smallest partition (0.007 Mbp) was too small to resolve by PFGE. The final assembly was based on $4946 \mathrm{Mbp}$ of Illumina draft data, which provided
Table 3 Summary of genome: one chromosome and five plasmids

\begin{tabular}{lllll}
\hline Label & Size (Mbp) & Topology & INSDC identifier & RefSeq ID \\
\hline Chromosome & 2.84 & circular & ATTJ01000001 & ATTJ01000001 \\
Megaplasmid 1 & 0.49 & circular & ATTJ010000002 & ATTJ01000002 \\
Megaplasmid 2 & 0.39 & circular & ATTJ01000003 & ATTJ01000003 \\
Megaplasmid 3 & 0.20 & unknown & ATTJ01000004 & ATTJ01000004 \\
Plasmid 1 & 0.098 & circular & ATTJ01000005 & ATTJ01000005 \\
Plasmid 2 & 0.007 & circular & ATTJ01000006 & ATTJ01000006 \\
\hline
\end{tabular}

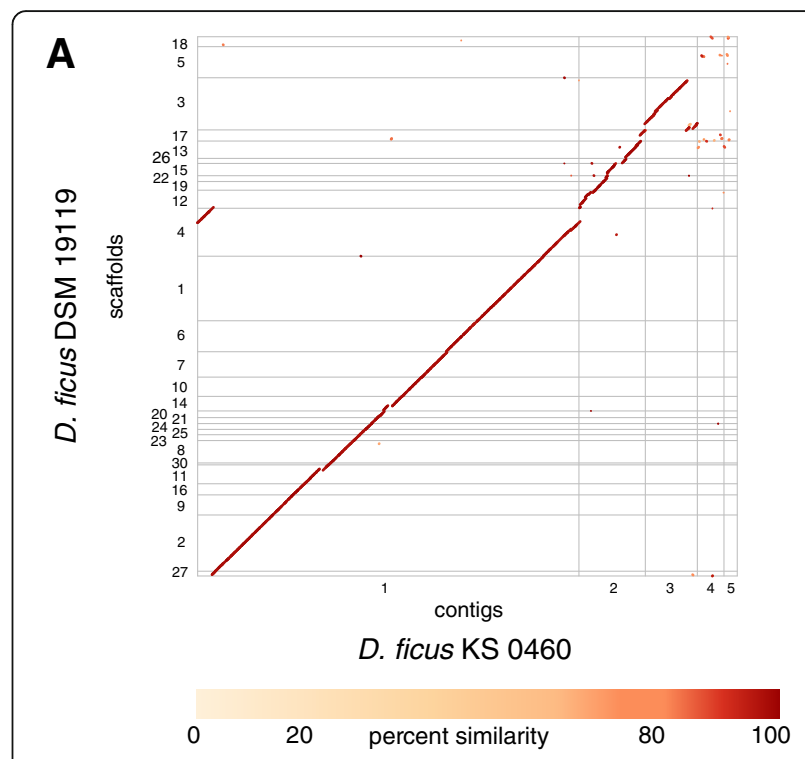

B

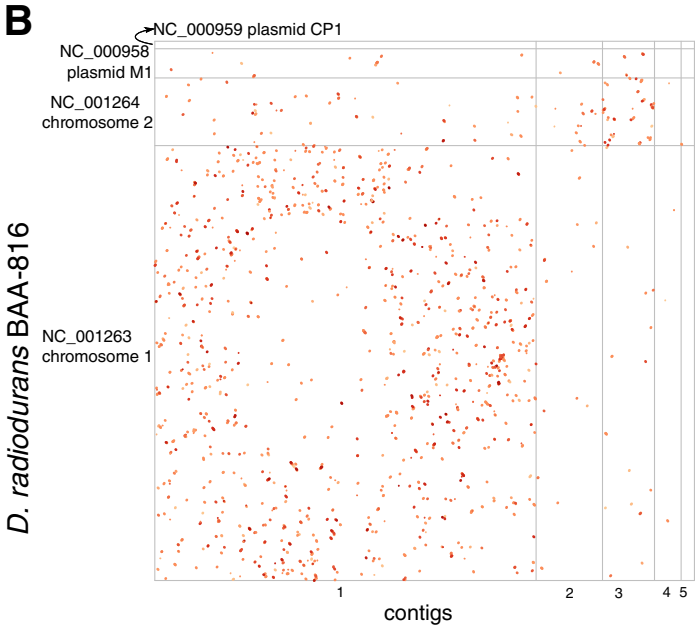

D. ficus KS 0460

Fig. 3 Genomic alignment of D. ficus KS 0460 with D. ficus DSM 19119 or D. radiodurans BAA-816. a Strain KS 0460 versus strain DSM 19119. b Strain KS 0460 versus strain BAA-816. Six-frame translations of scaffolds were aligned with Mummer 3.23. Homologous regions are plotted as dots, colored according to the similarity of the aligned loci. Diagonal lines of dots represent syntenic regions. Only contigs longer than $20 \mathrm{kbp}$ are shown. Axes are not drawn to scale 
an average of $1237 \times$ coverage of the genome. The total genomic GC content was $69.7 \%$ and was similar across all but the smallest contig, which contained $62.5 \%$ GC. The genome contains 3827 predicted protein-coding genes and 67 RNA-coding genes (total 3894).

\section{Insights from the genome sequence}

Comparative genomic analysis of strain KS 0460 confirmed the observations made on the basis of the 16S rDNA sequence (Fig. 1) - that the sequenced strain belongs to $D$. ficus and not to D. grandis, as originally reported. This is exemplified by the existence of long syntenic regions between the genomes of D. ficus strain KS 0460 and the type strain of D. ficus DSM 19119 (Fig. 3a), supporting near-identity between the strains; $16 \mathrm{~S}$ rDNA sequences of these two strains are 99\% identical. A close relationship between the strains is also supported by the high (97.8\%) genome-wide average nucleotide identity between the two genomes as well as the high (0.84) fraction of orthologous genes (alignment fraction) between them. The suggested cutoff values for average nucleotide identity and alignment fraction between genomes belonging to the same species are $96.5 \%$ and 0.60 , respectively [36]. The comparison between $D$. ficus KS 0460 and D. radiodurans BAA-816 revealed almost no synteny between these genomes (Fig. 3b). Approximately $76 \%$ of the predicted proteins contained identifiable Pfam domains, and $72 \%$ were assigned to COGs (Tables 4 and 5). Of all $D$. ficus KS 0460 proteins, 3059 and 2717 had homologues in D. radiodurans BAA-816 and D. geothermalis DSM 11300, respectively. Two regions with coordinates $150,375-159,184$ and 2,690,525-2,700,151 on the 2.84 Mbp chromosome [20] were identified as likely prophages of Myoviridae family using PHAST program

Table 4 Genome statistics

\begin{tabular}{lll}
\hline Attribute & Value & $\%$ of Total \\
\hline Genome size (bp) & $4,019,382$ & $100.00 \%$ \\
DNA coding (bp) & $3,614,725$ & $89.93 \%$ \\
DNA G + C (bp) & $2,803,041$ & $69.74 \%$ \\
DNA scaffolds & 6 & \\
Total genes & 3894 & $100.00 \%$ \\
Protein coding genes & 3827 & $98.28 \%$ \\
RNA genes & 67 & $1.72 \%$ \\
Pseudo genes & 45 & $1.16 \%$ \\
Genes in internal clusters & 982 & $25.66 \%$ \\
Genes with function prediction & 2831 & $72.7 \%$ \\
Genes assigned to CoGs & 2747 & $71.77 \%$ \\
Genes with Pfam domains & 2964 & $76.12 \%$ \\
Genes with signal peptides & 458 & $11.97 \%$ \\
Genes with transmembrane helices & 779 & $20.36 \%$ \\
CRISPR repeats & 0 & $0.00 \%$ \\
\hline
\end{tabular}

Table 5 Number of genes associated with general COG functional categories

\begin{tabular}{|c|c|c|c|}
\hline Code & Value & \%age & Description \\
\hline J & 226 & $6 \%$ & Translation, ribosomal structure and biogenesis \\
\hline A & 0 & $0 \%$ & RNA processing and modification \\
\hline K & 166 & $4 \%$ & Transcription \\
\hline L & 97 & $3 \%$ & Replication, recombination and repair \\
\hline B & 0 & $0 \%$ & Chromatin structure and dynamics \\
\hline D & 43 & $1 \%$ & $\begin{array}{l}\text { Cell cycle control, Cell division, chromosome } \\
\text { partitioning }\end{array}$ \\
\hline V & 71 & $2 \%$ & Defense mechanisms \\
\hline T & 228 & $6 \%$ & Signal transduction mechanisms \\
\hline M & 146 & $4 \%$ & Cell wall/membrane biogenesis \\
\hline N & 25 & $1 \%$ & Cell motility \\
\hline U & 23 & $1 \%$ & Intracellular trafficking and secretion \\
\hline O & 125 & $3 \%$ & $\begin{array}{l}\text { Posttranslational modification, protein } \\
\text { turnover, chaperones }\end{array}$ \\
\hline C & 152 & $4 \%$ & Energy production and conversion \\
\hline G & 179 & $5 \%$ & Carbohydrate transport and metabolism \\
\hline$E$ & 280 & $7 \%$ & Amino acid transport and metabolism \\
\hline $\mathrm{F}$ & 90 & $2 \%$ & Nucleotide transport and metabolism \\
\hline $\mathrm{H}$ & 149 & $4 \%$ & Coenzyme transport and metabolism \\
\hline । & 116 & $3 \%$ & Lipid transport and metabolism \\
\hline$P$ & 138 & $4 \%$ & Inorganic ion transport and metabolism \\
\hline Q & 58 & $2 \%$ & $\begin{array}{l}\text { Secondary metabolites biosynthesis, transport } \\
\text { and catabolism }\end{array}$ \\
\hline $\mathrm{R}$ & 217 & $6 \%$ & General function prediction only \\
\hline$S$ & 145 & $4 \%$ & Function unknown \\
\hline - & 1080 & $28 \%$ & Not in COGs \\
\hline
\end{tabular}

The total is based on the total number of protein coding genes in the genome. Proteins were assigned to the latest updated COG database using the COGnitor program [57]. Other functional categories: defense and mobilome account for $2 \%$ and $1 \%$, respectively

[37]. The largest number of transposable elements belongs to IS3 family (COG2801). There are 13 copies of this element in the genome. This transposon is absent in the genomes of $D$. radiodurans BAA-816 and D. geothermalis DSM 11300

\section{Extended insights}

The mapping of D. ficus KS 0460 genes to KEGG pathways by KOALA [38] showed that the strain contains the same DNA replication and repair genes as $D$. radiodurans, which were previously shown to be unremarkable [39] (Additional file 2: Table S1). The most striking differences between $D$. ficus KS 0460 and D. radiodurans BAA-816 identified by the comparison of the KEGG pathways were in purine degradation and nitrogen metabolism. Specifically, compared to $D$. radiodurans, $D$. ficus lacks guanine deaminase, xanthine dehydrogenase/oxidase, urate oxidase 5-hydroxyisourate hydrolase, 2-oxo-4-hydroxy-4-carboxy- 
5-ureidoimidazoline decarboxylase, allantoinase, allantoate deiminase, and the entire urease operon (DRA0311DRA0319 in D. radiodurans). In D. ficus KS 0460, these metabolic disruptions might contribute to the accumulation of $\mathrm{Mn}^{2+}$ antioxidants involved in the protection of proteins from radiation/desiccation-induced ROS [8]. In contrast, D. ficus KS 0460 contains eight genes involved in nitrogen metabolism, namely MFS transporter of NNP family, nitrate/nitrite transporter NarK, nitrate reductase/ nitrite oxidoreductase alpha subunit, nitrous oxideforming nitrite reductase, nitrous oxide reductase, nitrite reductase (cytochrome c-5 52), nitronate monooxygenase, hydroxylamine reductase $\mathrm{Hcp}$, and assimilatory nitrate reductase catalytic subunit NapA, that $D$. radiodurans BAA-816 lacks. Other genes present in D. ficus KS 0460 but absent in D. radiodurans BAA-816 are listed in Additional file 3: Table S2.

Despite the high intracellular $\mathrm{Mn}$ concentrations of Deinococcus species (Fig. 2f), one of the proteins missing in D. ficus KS 0460 is the homologue of the $D$. radiodurans nramp Mn-transporter (DR1709), previously identified as critical to extreme IR resistance [40, 41]. On the other hand, D. ficus KS 0460 encodes a manganese/ zinc/iron ABC transport system (KEGG Module M00319) that is also encoded in the $D$. radiodurans genome. This points to the existence of diverse genetic routes to the complex phenotype of extreme IR resistance even if the physico-chemical defense mechanisms (accumulation of $\mathrm{Mn}$ and small metabolites) may be the same [42].

The largest protein families expanded in D. ficus KS 0460 include several signal transduction proteins (e.g. CheY-like receiver domains, diguanylate cyclase, bacteriophytochrome-like histidine kinase), several families of acetyltransferases and a stress response protein DinB/ YfiT family (Fig. 4a). Many of these families are known to be specifically expanded in previously characterized Deinococcus species (Fig. 4b). Thus, D. ficus displays the same trend.

In addition to the nramp transporter, other genes previously considered to be important to IR resistance

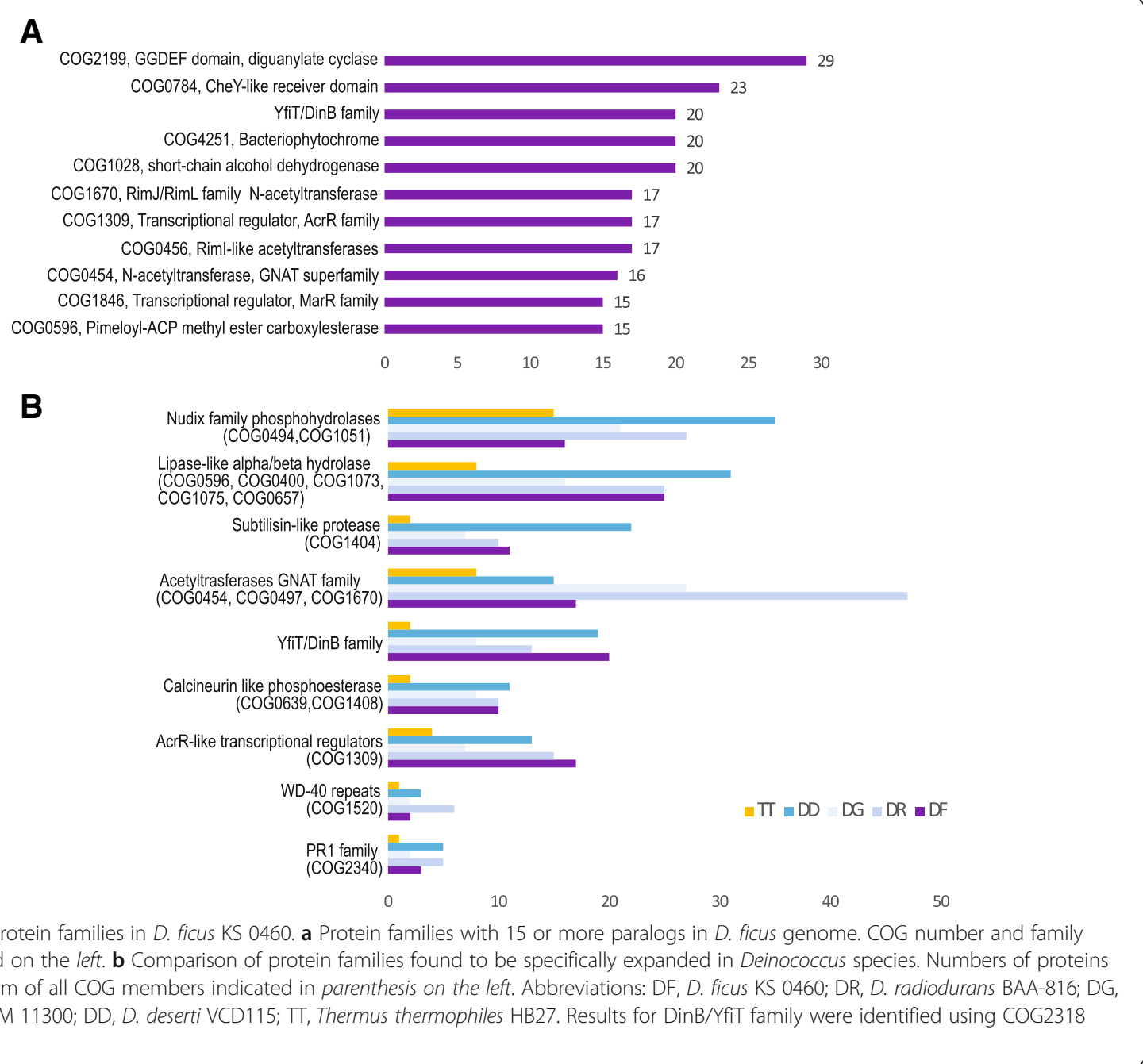


are missing in the genome of D. ficus KS 0460, namely, the proteins DdrF, DdrJ and DdrK, all of which are also missing in $D$. deserti $[3,40]$. DdrO and IrrE proteins found to be key players in regulation of irradiation responses in $D$. radiodurans and $D$. deserti $[43,44]$ are present in D. ficus KS 0460 (DeinoDRAFT_1503 and DeinoDRAFT_1002, respectively). This suggests that the same regulatory pathways are likely active in $D$. ficus KS 0460 .

\section{Conclusions}

Twenty years have passed since the extremely IR-resistant bacterium $D$. radiodurans became one of the first freeliving organisms to be subjected to whole genome sequencing [45]. Since then, comparative analyses between $D$. radiodurans and other high-quality draft and complete Deinococcus genomes have continued, but with few novel findings [10]. Deinococcus ficus KS 0460 hereby becomes the eleventh Deinococcus reference genome. We confirm by transmission electron microscopy that the very IRresistant strain KS 0460 grows as single bacillus-shaped cells, whereas deinococci typically grow as diplococci and tetracocci. Our $16 \mathrm{~S}$ rRNA phylogenetic analysis confirms that strain KS 0460 belongs to the genus Deinococcus, its ribosomal RNA being almost identical to the type strain of D. ficus DSM 19119. The D. ficus KS 0460 genome (4.019 Mbp) is $28 \%$ larger than D. radiodurans BAA816 and is divided into six genome partitions compared to four partitions in $D$. radiodurans. Of the 875 genes representing the core genome of ten Deinococcus species, only five genes are missing from $D$. ficus KS 0460. In other words, D. ficus KS 0460 exemplifies the Deinococcus lineage. In particular, D. ficus KS 0460 contains the same DNA replication and repair genes, and antioxidant genes (e.g. Mn-dependent superoxide dismutase and catalase) as $D$. radiodurans, which were previously shown to be unremarkable [10]. The most striking genomic differences between D. ficus KS 0460 and D. radiodurans BAA-816 are metabolic: (i) D. ficus lacks nine genes involved in purine degradation present in $D$. radiodurans, possibly contributing to the accumulation of small metabolites known to be involved in the production of $\mathrm{Mn}^{2+}$ antioxidants, which specifically protect proteins from IR-induced ROS; and (ii) D. ficus contains eight genes in nitrogen metabolism that are absent from $D$. radiodurans, including nitrate and nitrite reductases, suggesting that $D$. ficus has the ability to reduce nitrate, which could facilitate survival in anaerobic/microaerophilic environments. We also show that $D$. ficus KS 0460 accumulates high Mn concentrations and has a significantly higher antioxidant capacity than IR-sensitive bacteria. However, D. ficus KS 0460 lacks the homologue of the $D$. radiodurans nramp $\mathrm{Mn}$ transporter, previously identified as critical to extreme IR resistance [40, 41], but $D$. ficus $\mathrm{KS} 0460$ encodes at least one alternative manganese transport system. Thus, like previous Deinococcus genome comparisons, our D. ficus analysis demonstrates the limited ability of genomics to predict complex phenotypes, with the pool of genes consistently present in radioresistant, but absent from radiosensitive species of the phylum shrinking further $[3,10]$. With $D$. ficus KS 0460, the number of completed Deinococcus genomes is now sufficiently large to determine the core genome and pangenome of these remarkable bacteria. We anticipate that these fresh genomic insights will facilitate approaches applying Deinococcus $\mathrm{Mn}$ antioxidants in the production of irradiated vaccines $[46,47]$ and as in vivo radioprotectors [48].

\section{Additional files}

\begin{abstract}
Additional file 1: Figure S1. 165 rRNA phylogenetic tree of the Deinococcus genus. The multiple alignment of 165 rRNA sequences was constructed using MUSCLE program [58] with default parameters. The maximum-likelihood phylogenetic tree was reconstructed using the PHYML program [18], with GTR substitution matrix, empirical base frequencies, and gamma-distributed site rates; support values were computed using the aBayes method. Truepera radiovictrix was chosen as an outgroup. D. ficus KS 0460 is marked in red, D. ficus DSM 19119 in green, completely sequenced genomes (according to GenBank) in purple. (PDF $416 \mathrm{~kb}$ )
\end{abstract}

Additional file 2: Table S1. DNA repair genes that are present in $D$. ficus KS 0460 and in D. radiodurans BAA-816. (XLSX 13 kb)

Additional file 3: Table S2. Genes that are present in D. ficus KS 0460 but absent in D. radiodurans BAA-816. (XLSX $44 \mathrm{~kb}$ )

\section{Abbreviations}

COGs: Clusters of Orthologous Groups; D10: Dose yielding 10\% survival; IR: Ionizing radiation; KOALA: KEGG Orthology And Links Annotation; $\mathrm{Mn}^{2}$ ${ }^{+}$: Manganous ions; Net AUC: Net area under the fluorescence decay curve; PFGE: Pulsed-field gel electrophoresis; ROS: Reactive oxygen species; USUHS: Uniformed Services University of the Health Sciences

\section{Acknowledgements}

We thank Dr. Alexander Vasilenko for transmission electron microscopy of $D$. ficus KS 0460

\section{Funding}

The work performed at the Uniformed Services University of the Health Sciences was supported by a Defense Threat Reduction Agency grant HDTRA-18774-M. CG, NGC and TG acknowledge the support of the Slovenian Research Agency (BI-US/12-13-003, BI-US/14-15-009, Infrastructural Centre Mycosmo, MRIC UL) and the Research Program in Forest Biology, Ecology and Technology (P4-0107). The work conducted by the U.S. Department of Energy Joint Genome Institute, a DOE Office of Science User Facility, is supported by the Office of Science of the U.S. Department of Energy under Contract No. DE-AC02-05CH11231.

\section{Disclaimer}

The opinions expressed herein are those of the authors, and are not necessarily representative of those of the USUHS, the Department of Defense; or, the United States Army, Navy, or Air Force.

\section{Authors' contributions}

VM and EG designed experimental protocols; VM, EG, OG, PK, RT, RV and IC performed experiments, collection and analysis of data; MW was responsible for irradiator setup and dosimetry; EB purified the genomic DNA; CG, KM, TG, YW, $M H, A C, M P, K P, N V, N M, D S, T B K R, C D, N S, N I, N K, T W, H D, K D, T E, L G, W G, C M$, $H T, Y X$ and $P C$ were involved in sequencing, assembly, annotation and analysis of the D. ficus genome; CG, MD, KM, and VM drafted the manuscript; MD, EG, VM, OG, RT, GE, NG-C, TG and YW were involved in editing the final manuscript; and all authors read and approved the final manuscript. 


\section{Competing interests}

The authors declare they have no competing interests.

\section{Publisher's Note}

Springer Nature remains neutral with regard to jurisdictional claims in published maps and institutional affiliations.

\section{Author details}

${ }^{1}$ Uniformed Services University of the Health Sciences, School of Medicine, Bethesda, MD, USA. ${ }^{2}$ Henry M. Jackson Foundation for the Advancement of Military Medicine, Bethesda, MD, USA. ${ }^{3}$ National Center for Biotechnology Information, National Library of Medicine, National Institutes of Health, Bethesda, MD, USA. ${ }^{4}$ University of Bielefeld, Bielefeld, Germany. ${ }^{5}$ Leibniz Institute DSMZ - German Collection of Microorganisms and Cell Cultures, Braunschweig, Germany. ${ }^{6}$ DOE Joint Genome Institute, Walnut Creek, CA, USA. ${ }^{7}$ Los Alamos National Laboratory, Los Alamos, NM, USA. ${ }^{8}$ Department of Biology, Biotechnical Faculty, University of Ljubljana, Ljubljana, Slovenia.

${ }^{9}$ Slovenian Forestry Institute, Ljubljana, Slovenia.

\section{Received: 9 March 2017 Accepted: 20 July 2017}

\section{Published online: 28 July 2017}

\section{References}

1. Daly MJ. A new perspective on radiation resistance based on Deinococcus radiodurans. Nat Rev Microbiol. 2009; doi:10.1038/nrmicro2073.

2. Ludwig W, Strunk O, Westram R, Richter L, Meier H, et al. ARB: a software environment for sequence data. Nucleic Acids Res. 2004; doi:10.1093/nar/ gkh293.

3. Makarova KS, Daly MJ. Comparative genomics of stress response systems in Deinococcus bacteria. In: Storz G, Hennge R, editors. Bacterial stress responses; 2011. p. 445-57

4. Oyaizu H, Stackebrandt E, Schleifer KH, Ludwig W, Pohla H, Ito H, et al. A radiation-resistant rod-shaped bacterium, Deinobacter grandis gen. nov., sp. nov., with peptidoglycan containing ornithine. Int J Syst Bacteriol. 1987;37:62-7.

5. Rainey FA, Nobre MF, Schumann P, Stackebrandt E, Da Costa MS Phylogenetic diversity of the deinococci as determined by $16 \mathrm{~S}$ ribosomal DNA sequence comparison. Int J Syst Bacteriol. 1997; doi:10. 1099/00207713-47-2-510.

6. Daly MJ. Engineering radiation-resistant bacteria for environmental biotechnology. Curr Opin Biotechnol. 2000;11(3):280-5.

7. Daly MJ, Gaidamakova EK, Matrosova VY, Vasilenko A, Zhai M, Venkateswaran $A$, et al. Accumulation of $\mathrm{Mn}(\mathrm{II})$ in Deinococcus radiodurans facilitates gammaradiation resistance. Science. 2004; doi:10.1126/science.1103185.

8. Daly MJ. Death by protein damage in irradiated cells. DNA Repair (Amst.) 2012; doi:10.1016/j.dnarep.2011.10.024.

9. Lai WA, Kampfer P, Arun AB, Shen FT, Huber B, Rekha PD, Young CC. Deinococcus ficus sp. nov., isolated from the rhizosphere of Ficus religiosa $\mathrm{L}$. Int J Syst Evol Microbiol. 2006; doi:10.1099/ijs.0.64007-0.

10. Makarova KS, Omelchenko MV, Gaidamakova EK, Matrosova VY, Vasilenko A, Zhai $M$, et al. Deinococcus geothermalis: the pool of extreme radiation resistance genes shrinks. PLoS One. 2007; doi:10.1371/journal.pone.0000955.

11. Slade D, Radman M. Oxidative stress resistance in Deinococcus radiodurans. Microbiol Mol Biol Rev. 2011; doi:10.1128/MMBR.00015-10

12. Brim H, McFarlan SC, Fredrickson JK, Minton KW, Zhai M, Wackett LP, Daly MJ. Engineering Deinococcus radiodurans for metal remediation in radioactive mixed waste environments. Nat Biotechnol. 2000; doi:10.1038/71986.

13. Daly MJ, Gaidamakova EK, Matrosova VY, Kiang JG, Fukumoto R, Lee DY, et al. Small-molecule antioxidant proteome-shields in Deinococcus radiodurans. PLoS One. 2010; doi:10.1371/journal.pone.0012570.

14. Sun H, Li M, Xu G, Chen H, Jiao J, Tian B, et al. Regulation of MntH by a dual $\mathrm{Mn}(\mathrm{II})$ - and $\mathrm{Fe}(\mathrm{II})$-dependent transcriptional repressor (DR2539) in Deinococcus radiodurans. PLoS One. 2012; doi:10.1371/journal.pone.0035057.

15. Daly MJ, Gaidamakova EK, Matrosova VY, Vasilenko A, Zhai M, Leapman RD, Fredrickson JK. Protein oxidation implicated as the primary determinant of bacterial radioresistance. PLoS Biol. 2007; doi:10.1371/journal.pbio.0050092.

16. Ivanova N, Rohde C, Munk C, Nolan M, Lucas S, Del Rio TG, et al. Complete genome sequence of Truepera radiovictrix type strain (RQ-24). Stand Genomic Sci. 2011; doi: 10.4056/sigs.1563919

17. Price MN, Dehal PS, Arkin AP. FastTree 2 - approximately maximumlikelihood trees for large alignments. PLoS One 2010; doi: 10.1371/journal. pone.0009490.
18. Guindon S, Gascuel O. A simple, fast, and accurate algorithm to estimate large phylogenies by maximum likelihood. Syst Biol. 2003;52(5):696-704.

19. JGI Genome Portal Deinococcus sp. 2009 database. http://genome.jgi.doe.gov/ DeinoDRAFT_10292/DeinoDRAFT_10292.info.html. Accessed 9 May 2017.

20. NCBI GenBank site. https://www.ncbi.nlm.nih.gov/nuccore/ATTJ00000000.1. Accessed 9 May 2017.

21. Simao FA, Waterhouse RM, loannidis P, Kriventseva EV, Zdobnov EM. BUSCO: assessing genome assembly and annotation completeness with single-copy orthologs. Bioinformatics. 2015; doi:10.1093/bioinformatics/btv351.

22. Contreras-Moreira B, Vinuesa P. GET_HOMOLOGUES, a versatile software package for scalable and robust microbial pangenome analysis. Appl Environ Microbiol. 2013; doi:10.1128/AEM.02411-13.

23. Bennett S. Solexa Ltd. Pharmacogenomics. 2004; doi:10.1517/14622416.5.4.433.

24. Zerbino DR, Birney E. Velvet: algorithms for de novo short read assembly using de Bruijn graphs. Genome Res. 2008; doi:10.1101/gr.074492.107.

25. Ewing B, Green P. Base-calling of automated sequencer traces using phred. II Error probabilities Genome Res. 1998:8(3):186-94.

26. Ewing B, Hillier L, Wendl MC, Green P. Base-calling of automated sequencer traces using phred. I Accuracy assessment Genome Res. 1998;8(3):175-85.

27. Gordon D, Abajian C, Green P. Consed: a graphical tool for sequence finishing. Genome Res. 1998:8(3):195-202.

28. Huntemann M, Ivanova NN, Mavromatis K, Tripp HJ, Paez-Espino D, Palaniappan $\mathrm{K}$, et al. The standard operating procedure of the DOE-JGI microbial genome annotation pipeline (MGAP v.4). Stand Genomic Sci. 2015; doi:10.1186/s40793-015-0077-y.

29. Markowitz VM, Mavromatis K, Ivanova NN, Chen IM, Chu K, Kyrpides NC. IMG ER: a system for microbial genome annotation expert review and curation. Bioinformatics. 2009; doi:10.1093/bioinformatics/btp393.

30. Hyatt D, Chen GL, Locascio PF, Land ML, Larimer FW, Hauser LJ. Prodigal: prokaryotic gene recognition and translation initiation site identification. BMC Bioinformatics 2010; doi:10.1186/1471-2105-11-119.

31. Pati A, Ivanova NN, Mikhailova N, Ovchinnikova G, Hooper SD, Lykidis A Kyrpides NC. GenePRIMP: a gene prediction improvement pipeline for prokaryotic genomes. Nat Methods 2010; doi:10.1038/nmeth.1457.

32. Chen IA, Markowitz VM, Chu K, Palaniappan K, Szeto E, Pillay M, et al. IMG/ $M$ : integrated genome and metagenome comparative data analysis system. Nucleic Acids Res. 2016; doi:10.1093/nar/gkw929.

33. Altschul SF, Gish W, Miller W, Myers EW, Lipman DJ. Basic local alignment search tool. J Mol Biol. 1990; doi:10.1016/S0022-2836(05)80360-2.

34. Petersen TN, Brunak S, von Heijne G, Nielsen H. SignalP 4.0: discriminating signal peptides from transmembrane regions. Nat Methods. 2011; doi:10. 1038/nmeth.1701.

35. Sonnhammer EL, von Heijne G, Krogh A. A hidden Markov model for predicting transmembrane helices in protein sequences. Proc Int Conf Intell Syst Mol Biol. 1998;6:175-82.

36. Varghese NJ, Mukherjee S, Ivanova N, Konstantinidis KT, Mavrommatis K, Kyrpides NC, Pati A. Microbial species delineation using whole genome sequences. Nucleic Acids Res. 2015; doi:10.1093/nar/gkv657.

37. Zhou Y, Liang Y, Lynch KH, Dennis JJ, Wishart DS. PHAST: a fast phage search tool. Nucleic Acids Res. 2011; doi:10.1093/nar/gkr485.

38. Kanehisa M, Sato Y, Morishima K. BlastKOALA and GhostKOALA: KEGG tools for functional characterization of genome and metagenome sequences. J Mol Biol. 2016; doi:10.1016/j.jmb.2015.11.006

39. Makarova KS, Aravind L, Wolf Yl, Tatusov RL, Minton KW, Koonin EV, Daly MJ. Genome of the extremely radiation-resistant bacterium Deinococcus radiodurans viewed from the perspective of comparative genomics. Microbiol Mol Biol Rev. 2001; doi:10.1128/MMBR.65.1.44-79.2001.

40. Liu Y, Zhou J, Omelchenko MV, Beliaev AS, Venkateswaran A, Stair J, et al. Transcriptome dynamics of Deinococcus radiodurans recovering from ionizing radiation. Proc Natl Acad Sci U S A. 2003; doi:10.1073/pnas.0630387100.

41. Chang S, Shu H, Li Z, Wang Y, Chen L, Hua Y, Qin G. Disruption of manganese ions $[\mathrm{Mn}(\mathrm{II})]$ transporter genes DR1709 or DR2523 in extremely radio-resistant bacterium Deinococcus radiodurans. Wei Sheng Wu Xue Bao. 2009:49(4):438-44

42. Culotta VC, Daly MJ. Manganese complexes: diverse metabolic routes to oxidative stress resistance in prokaryotes and yeast. Antioxid Redox Signal. 2013; doi:10.1089/ars.2012.5093

43. Devigne A, Ithurbide S, Bouthier de la Tour, Passot F, Mathieu M, Sommer S, Servant P. DdrO is an essential protein that regulates the radiation desiccation response and the apoptotic-like cell death in the radioresistant Deinococcus radiodurans bacterium. Mol Microbiol. 2015; doi:10.1111/mmi.12991. 
44. Ludanyi M, Blanchard L, Dulermo R, Brandelet G, Bellanger L, Pignol D, Lemaire D, de Groot A. Radiation response in Deinococcus deserti: IrrE is a metalloprotease that cleaves repressor protein DdrO. Mol Microbiol. 2014; doi:10.1111/mmi.12774.

45. White O, Eisen JA, Heidelberg JF, Hickey EK, Peterson JD, Dodson RJ, et al. Genome sequence of the radioresistant bacterium Deinococcus radiodurans R1. Science. 1999;286(5444):1571-7.

46. Gaidamakova EK, Myles IA, McDaniel DP. Fowler CJ, Valdez PA, Naik S, et al. Preserving immunogenicity of lethally irradiated viral and bacterial vaccine epitopes using a radio- protective Mn2+-peptide complex from Deinococcus. Cell Host Microbe. 2012; doi:10.1016/j.chom.2012.05.011.

47. Gayen M, Gupta P, Morazzani EM, Gaidamakova EK, Knollmann-Ritschel B, Daly MJ, et al. Deinococcus Mn2+-peptide complex: a novel approach to alphavirus vaccine development. Vaccine. 2017; doi:10.1016/j.vaccine.2017.05.016.

48. Gupta P, Gayen M, Smith JT, Gaidamakova EK, Matrosova VY, Grichenko O, et al. MDP: a Deinococcus Mn2+-decapeptide complex protects mice from ionizing radiation. PLoS One 2016; doi:10.1371/journal.pone.0160575.

49. Field D, Garrity G, Gray T, Morrison N, Selengut J, Sterk P, et al. The minimum information about a genome sequence (MIGS) specification. Nat Biotechnol. 2008; doi:10.1038/nbt1360.

50. Woese CR, Kandler O, Wheelis ML. Towards a natural system of organisms: proposal for the domains Archaea, Bacteria, and Eucarya. Proc Natl Acad Sci U S A. 1990:87(12):4576-9.

51. Weisburg WG, Giovannoni SJ, Woese CR. The Deinococcus-Thermus phylum and the effect of rRNA composition on phylogenetic tree construction. Syst Appl Microbiol. 1989;11:128-34.

52. Garrity GM, Holt JG. Taxonomic outline of the Archaea and Bacteria. In: Garrity GM, Boone DR, Castenholz RW, editors. Bergey's manual of systematic bacteriology. Second edition, volume 1. New York: Springer; 2001. p. 155-66.

53. List Editor. Validation list no. 85. Validation of publication of new names and new combinations previously effectively published outside the IJSEM. Int J Syst Evol Microbiol. 2002;52:685-90. http://dx.doi.org/10.1099/ijs.0.63767-0.

54. Garrity GM, Holt JG. Class I. In: Garrity GM, Boone DR, Castenholz RW, editors. Bergey's manual of systematic bacteriology. Second edition, volume 1. New York: Springer; 2001. p. 395.

55. Brooks BW, Murray RG. Nomenclature for "Micrococcus radiodurans" and other radiation-resistant cocci: Deinococcaceae fam. nov. and Deinococcus gen. nov., including five species. Int J Syst Bacteriol. 1981; doi:10.1099/00207713-31-3-353.

56. Ashburner M, Ball CA, Blake JA, Botstein D, Butler H, Cherry JM, et al. Gene ontology: tool for the unification of biology. The gene ontology consortium. Nat Genet. 2000;25:25-9.

57. Galperin MY, Makarova KS, Wolf YI, Koonin EV. Expanded microbial genome coverage and improved protein family annotation in the COG database. Nucleic Acids Res. 2015; doi:10.1093/nar/gku1223.

58. Edgar RC. MUSCLE: a multiple sequence alignment method with reduced time and space complexity. BMC Bioinformatics. 2004; doi:10.1186/1471-2105-5-113.

59. Price MN, Dehal PS, Arkin AP. FastTree: computing large minimum evolution trees with profiles instead of a distance matrix. Mol. Biol. Evol. 2009; doi:10. 1093/molbev/msp077.

60. Ghosal D, Omelchenko MV, Gaidamakova EK, Matrosova VY, Vasilenko A, Venkateswaran A, et al. How radiation kills cells: survival of Deinococcus radiodurans and Shewanella oneidensis under oxidative stress. FEMS Microbiol. Rev. 2005; doi:10.1016/j.fmrre.2004.12.007.

61. Fredrickson JK, Zachara JM, Balkwill DL, Kennedy D, Li SM, Kostandarithes $\mathrm{HM}$, et al. Geomicrobiology of high-level nuclear waste-contaminated vadose sediments at the Hanford site, Washington State. Appl Environ Microbiol. 2004; doi: 10.1128/AEM.70.7.4230-4241.2004.

62. Masters $\mathrm{Cl}$, Murray RG, Moseley BE, Minton KW. DNA polymorphisms in new isolates of 'Deinococcus radiopugnans'. J Gen Microbiol. 1991; doi: 10.1099/ 00221287-137-7-1459.

63. Dávalos A, Bartolomé B, Suberviola J, Gómez-Cordovés C. ORAC- fluorescein as a model for evaluating antioxidant activity of wines. Pol J Food Nutr Sci. 2003;12(53):133-6.

64. Mikami I, Yamaguchi M, Shinmoto $H$, Tsushida T. Development and validation of a microplate-based $\beta$-carotene bleaching assay and comparison of antioxidant activity (AOA) in several crops measured by $\beta$-carotene bleaching, DPPH and ORAC assays. Food Sci Technol Res. 2009; 15(2):171-8

\section{Submit your next manuscript to BioMed Central and we will help you at every step:}

- We accept pre-submission inquiries

- Our selector tool helps you to find the most relevant journal

- We provide round the clock customer support

- Convenient online submission

- Thorough peer review

- Inclusion in PubMed and all major indexing services

- Maximum visibility for your research

Submit your manuscript at www.biomedcentral.com/submit

) Biomed Central 